\title{
Tamaño de población y parámetros repro- ductores del halcón peregrino Falco peregri- nus en Álava (País Vasco, España). Año 2016.
} Population size and reproductive parameters of the Peregrine falcon Falco peregrinus in Álava (Basque Country, Spain). Year 2016.

José Antonio Gainzarain ${ }^{1 *}$, Gorka Belamendia² ${ }^{2}$ Juan Manuel Pérez de $\mathrm{Ana}^{3}$

\section{Resumen}

En 2016 se censó la población reproductora de halcón peregrino Falco peregrinus en la provincia de Álava, manteniendo la periodicidad de diez años entre censos de ocasiones anteriores. Con una metodología estandarizada que concreta el número de visitas a cada territorio y la duración de las mismas, se localizaron 31 parejas territoriales, una cifra que se ha mantenido muy constante en los censos de los últimos veinte años. Los territorios con una ocupación más continuada a lo largo de este periodo son aquellos correspondientes a roquedos con una elevada dominancia sobre el terreno circundante. Tras los malos resultados reproductores observados diez años atrás, en 2016 se recuperan unos valores de productividad relativamente elevados, 1,40 pollos volados/ pareja territorial, aunque la elevada proporción de hembras inmaduras (17\%) podría indicar una alta mortalidad adulta.

Palabras clave: censo, evolución poblacional, éxito reproductor, hembras inmaduras.

\footnotetext{
1 Instituto Alavés de la Naturaleza Apdo. de correos 2092. 01080 Vitoria-Gasteiz

2 Centro de Estudios Ambientales. Ayuntamiento de Vitoria-Gasteiz Casa Dehesa de Olarizu s/n. 01006 Vitoria-Gasteiz

3 Diputación Foral de Bizkaia. Departamento de Sostenibilidad y Medio Natural. Lehendakari Aguirre, 9 - 2. 48014 Bilbao 


\begin{abstract}
In 2016, a census was carried out of the population of Peregrine Falcon Falco peregrinus in the province of Álava, maintaining the the periodicity of ten years between consecutive surveys applied on previous occasions. With a standardized methodology which established the number of visits to each territory and their duration, 31 territorial pairs were detected. This figure indicates a remarkable population stability over the last two decades. The territories with a more continuous occupancy pattern during this period were those corresponding to cliffs with a commanding position over the surrounding terrain. A productivity of 1.40 fledged young/ territorial pair was obtained in 2016, a remarkable recovery from the low breeding success observed ten years earlier. The most concerning aspect of the present census was the substantial proportion of immature females forming part of a pair (17\%), which may be a sign of high adult mortality.
\end{abstract}

Key words: census, immature females, population trend, reproductive success.

\title{
Laburpena
}

Aurrekoetan egindako zentsuetan bezala hamar urteko aldizkakotasunari eutsiz, 2016an Arabako belatz handiaren populazio ugaltzailearen zentsua egin zen. Eremu bakoitzera egin beharreko bisiten kopurua eta iraupena zehazten duen metodologia estandarizatuari jarraituz, 31 bikote eremutar aurkitu ziren; kopuru hau azken hogei urteetako zentsuetan nahikoa egonkor mantendu da. Denbora epe honetan, tarte luzeagoan okupatuta egon diren lurraldeak inguruko eremuarekiko dominantzia altua duten haitz-eremuak dira. Duela hamar urte ugalketa ez zen batere arrakastatsua izan; baina 2016an, ordea, berriro ere nahiko produktibitate balio altuak behatu ditugu: 1.40 txita hegaz/eremuko bikote bakoitzeko. Dena den, eme gazteen proportzio altuak (\%17a), helduen hilkortasun altua adieraz lezake.

Gako hitzak: zentsua, populazioaren eboluzioa, ugaltze-arrakasta, eme gazteak.

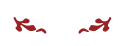

\section{Introducción}

El seguimiento a largo plazo de la evolución poblacional de una determinada especie resulta una herramienta de gran utilidad para la detección de amenazas que puedan comprometer su conservación (Tellería, 2012). Entre los grupos animales que con mayor frecuencia han sido objeto de este tipo de estudios destacan las aves rapaces diurnas (orden Falconiformes) (Hardey et al., 2009) y, entre ellas, el halcón peregrino (Falco peregrinus Tunstall, 1771), especie para la que se cuenta con un detallado 
conocimiento del estado de sus poblaciones, al menos en gran parte de Europa y Norteamérica (Cade et al., 1988; Rattcliffe, 1993; Zuberogoitia et al., 2002; Sielicki \& Mizera, 2009).

Esta especie presenta una distribución cosmopolita y se reparte ampliamente por casi toda Europa, con unos efectivos en España en el año 2008 estimados en 2462-2804 parejas (del Moral \& Molina, 2009). En el País Vasco los primeros datos sobre el tamaño de población de la especie corresponden al atlas de vertebrados continentales de los años 80 (Álvarez et al., 1985). En esta publicación se estimaron unos efectivos de 25-35 parejas para toda la comunidad autónoma, lo que con toda probabilidad constituye una notable subestimación del tamaño de población real (Zuberogoitia, 1997). Esta cifra se incluyó en la primera estima nacional, que situó la población española en 1628-1751 parejas (Heredia et al., 1988). Ya en los primeros años del presente siglo, datos más precisos sitúan la población vasca en 93-97 parejas reproductoras (Gainzarain et al., 2002), mientras que el censo nacional de 2008 (del Moral \& Molina, 2009) eleva esta cifra a 101. Esto equivale a una densidad de 1,42 parejas $/ 100 \mathrm{~km}^{2}$, una de las más elevadas de la España peninsular, tan solo superada por la comunidad autónoma de Cantabria (del Moral \& Molina, 2009).

En Álava la población de halcón peregrino fue censada en 1996/97, localizándose 31 33 parejas, con una productividad de 1,44 pollos/pareja territorial (Gainzarain et al., 2000). Posteriormente, durante seis años consecutivos (2000-2005), se llevó a cabo un seguimiento de la reproducción de una muestra de territorios, con unos valores de productividad que oscilaron entre 0,67 y 1,55 (promedio de 1,12; datos propios). El censo se repitió entre los años 2006 y 2007, con un resultado de 31 parejas, (del Moral \& Molina, 2009), y entre 2006 y 2008 se efectúo asimismo el seguimiento de la reproducción en una serie de territorios, que arrojó unos valores de productividad inferiores a los de años precedentes, con una media de los tres años de 0,67 pollos/pareja (del Moral \& Molina, 2009).

Con la intención de mantener la periodicidad de diez años entre censos sucesivos, en 2016 se llevó a cabo un nuevo conteo de los territorios ocupados por esta especie en Álava. Utilizando una metodología semejante a la de estudios precedentes, el objetivo de este nuevo censo es obtener información actualizada y precisa sobre el número de parejas nidificantes de halcón peregrino en la provincia, su distribución y sus tasas reproductoras. Los resultados obtenidos a lo largo de los diversos años de seguimiento se han empleado asimismo para analizar la relación entre la productividad de la población y la precipitación total en abril, mes en el que nacen los pollos y en el que son por tanto más vulnerables ante el mal tiempo (Horne \& Fielding, 2002; Zuberogoitia et al., 2002). Finalmente, con los datos de tres censos repartidos en veinte años, se pretende también investigar la relación entre la constancia de ocupación de los diversos territorios y las características ambientales de los mismos. 


\section{Métodos}

\section{Área de estudio, metodología de censo, y estima de la productividad}

El presente estudio se llevó a cabo en la provincia de Álava $\left(3.037 \mathrm{~km}^{2}\right)$ y el enclave burgalés de Treviño, con una superficie conjunta de $3.317 \mathrm{~km}^{2}$ y una altitud máxima de $1481 \mathrm{~m}$ s.n.m. Esta área presenta en su mayor parte un clima de transición atlántico-mediterráneo, y su relieve se caracteriza por amplios valles dedicados a la agricultura y ganadería, separados por sierras de mediana altitud, muchas de ellas con abundancia de roquedos calizos. El trabajo de campo se desarrolló durante la temporada de cría de 2016 y se dividió en dos partes, correspondientes a sendos periodos del ciclo reproductor de la especie, que han servido para conocer tanto el tamaño poblacional como las tasas reproductoras de la población alavesa.

La primera parte del estudio se desarrolló entre el 15 de febrero y el 21 de marzo, época en la que la detección de las parejas es sencilla, al estar estas muy aquerenciadas al roquedo de nidificación y presentar conspicuos comportamientos territoriales y de cortejo (Ratcliffe, 1993). Además, tras este periodo, las parejas que no inician la reproducción o fracasan en una fase temprana de la misma resultan mucho más difíciles de detectar, por lo que un censo en fechas más tardías es probable que las pasara por alto, lo que supondría una subestimación de los efectivos totales.

Se visitaron un total de 47 territorios con nidificación conocida en los últimos veinte años, y otros ocho más con aparentes buenas condiciones para el asentamiento de la especie (Gainzarain et al., 2000). Estos territorios, visitados también en censos precedentes con un esfuerzo de muestreo comparable, se prospectaron con ayuda de prismáticos y telescopio en las fechas señaladas anteriormente. Las visitas finalizaron bien con la detección de la pareja de halcones, bien tras noventa minutos de observación (un periodo de tiempo similar al empleado en censos anteriores). Con la pareja detectada, el territorio se daba como ocupado y no se volvía a visitar en esta fase del estudio. Si las condiciones de observación lo permitían, se anotó la edad de los componentes de la pareja (ave con plumaje de adulto/ ave de segundo año calendario). Si no se observaban halcones, o se detectaba un solo individuo, la visita se repetía siguiendo el mismo protocolo tras un intervalo mínimo de siete días. Asimismo, se llevaron a cabo visitas adicionales en el caso de que en alguna de las dos primeras la visibilidad del roquedo fuera deficiente, debido a la meteorología adversa. En resumen, para cada territorio potencial el resultado de esta fase del estudio podía estar entre alguna de estas opciones:

- Ocupado: observada una pareja de la especie.

- No ocupado: Ningún halcón detectado, o un solo ejemplar observado, tras dos periodos de observación de noventa minutos en días separados al menos por una semana y en buenas condiciones de visibilidad. 
La segunda fase del estudio se llevó a cabo a partir del 15 de mayo y sirvió para conocer el resultado de la reproducción de una serie de parejas representativas. Para ello se seleccionaron al azar veinte parejas del total localizado en la primera fase del estudio. Esta muestra fue objeto de seguimiento con el fin de conocer cuántas de ellas finalizaron con éxito la cría y cuántos pollos volaron del nido. Los territorios fueron visitados hasta que se localizaron los pollos, bien ya volando, bien aún en el nido, pero totalmente emplumados y a punto de emprender el vuelo. Para certificar el fracaso en la reproducción, y con un criterio similar al empleado en la primera fase del estudio, se precisaron tres visitas de dos horas de duración sin que se observaran pollos ni indicios de nido ocupado. Las tasas reproductoras calculadas fueron las de éxito reproductor (porcentaje de parejas territoriales que sacan adelante al menos un pollo), productividad ( $n^{\circ}$ de pollos volados por pareja territorial) y tasa de vuelo $\left(n^{\circ}\right.$ de pollos volados por pareja con éxito).

\section{Variables ambientales}

Con los datos de tres censos en un periodo de veinte años se analizó el papel de una serie de variables ambientales en la ocupación permanente o esporádica de un territorio determinado. Con este fin, se separaron, por un lado, los territorios con ocupación en los tres censos de aquellos regentados por una pareja en solo uno de ellos. Se excluyeron aquellos ocupados en dos de los tres censos con el objetivo de maximizar las diferencias entre grupos en la constancia de ocupación. Se excluyó asimismo un territorio correspondiente a una pequeña pared en el norte de la provincia, ocupada en 2016 pero que no se visitó en censos anteriores y que, por tanto, no puede encuadrarse en el grupo de las ocupadas en un solo censo, dado que se desconoce en realidad su situación en los dos primeros.

Para cada uno de los territorios se consideraron las siguientes variables ambientales, cuya relación con la selección de hábitat de la especie se ha señalado en estudios precedentes (Gainzarain et al., 2000; Sergio et al., 2004; Wightman \& Fuller, 2005; Rodríguez et al., 2007):

- Altitud en m s.n.m. de la parte superior del roquedo ocupado.

- Altura máxima del roquedo ocupado.

- Dominancia del roquedo ocupado, calculada como el promedio de la diferencia de altura entre la parte superior del mismo y el extremo de tres líneas imaginarias de $1 \mathrm{~km}$ que se proyectan hacia el frente formando un ángulo de $90^{\circ}$ y su bisectriz (Gainzarain et al., 2000).

- Cobertura del bosque en un radio de $2 \mathrm{~km}$.

- Cobertura de cultivos y prados de siega en un radio de $2 \mathrm{~km}$.

- Distancia a la carretera más cercana. 
- Distancia al núcleo de población más cercano.

- Distancia al nido de Águila real Aquila chrysaetos más cercano (ocupado al menos una vez con posterioridad a 1996, el primer año de censo) (datos propios).

Todas las variables se midieron en m, a excepción de las coberturas, expresadas en porcentaje. La altura del roquedo se estimó directamente sobre el terreno, la cobertura de bosques y de cultivos mediante el programa ArcGis 10.4.1 (Esri, 2016) a partir de los mapas de cobertura del suelo Corine Land Cover (fuente: http://centrodedescargas.cnig.es/CentroDescargas/ index.jsp), y el resto de variables mediante el programa Google Earth (https://www.google.com/intl/es/earth/).

Con el fin de analizar la relación entre la productividad de la población estudiada y la precipitación total en abril se utilizaron los datos correspondientes a la estación meteorológica de Arkaute, situada en el centro de Álava. Estos valores pueden considerarse representativos de todo el territorio, al hallarse fuertemente correlacionados (periodo 2001-16) con los obtenidos en las estaciones de Saratxo, en el norte de la provincia, $\left(r_{S}=0,803, n=16, P<0,001\right)$ y de Herrera, en el $\operatorname{sur}\left(r_{S}=0,826, n=16, P<0,001\right)$ (fuente: www.euskalmet.euskadi.eus).

\section{Análisis de datos}

Para determinar las características del hábitat asociadas a la continuidad de la ocupación de los territorios de halcón peregrino se elaboró un modelo lineal generalizado (GLM), en el que se utilizaron como predictores las variables ambientales descritas anteriormente. La variable dicotómica resultante de dividir los territorios entre los ocupados durante los tres censos de la especie en Álava y los ocupados en solo uno de ellos se empleó como variable dependiente, considerando una distribución binomial con función de vínculo logit (Zuur et al., 2007). Dado que varias de las variables predictoras se hallan fuertemente correlacionadas entre sí, para evitar el efecto de esta colinealidad se calculó el coeficiente de correlación entre todas ellas, y, siguiendo a Dormann et al. (2013), de cada par de variables con $r>0,7$ se eliminó aquella con una relación más débil con la variable dependiente (calculada mediante el correspondiente GLM de distribución binomial con una única variable predictora). Tras esta selección previa, la inclusión de variables en el modelo final se llevó a cabo mediante una selección hacia atrás por pasos, eliminando sucesivamente la variable con una menor contribución al modelo hasta que todas las incluidas en este fueran estadísticamente significativas.

La relación entre los resultados de la reproducción y la meteorología primaveral se abordó mediante un análisis de correlación de Spearman entre la productividad de la población en los años con datos y la precipitación total en abril. Los datos de productividad en 1996-97 corresponden a Gainzarain et al. (2000), los de 2006-2008 a del Moral \& 
Molina (2009), mientras que los del sexenio 2000-05 son datos propios no publicados, todos ellos obtenidos con una metodología similar a la de 2016. Para este y los anteriores análisis estadísticos se utilizó el programa R versión 3.5.0 (R Core Team, 2018).

\section{Resultados}

\section{Tamaño y distribución de la población}

Se localizaron un total de 31 parejas de la especie, treinta de ellas en Álava y una en el condado de Treviño. Además, en un territorio compartido con la provincia de Burgos, la pareja establecida crio en 2016 en la parte burgalesa, por lo que no se contabilizó en los resultados totales. No se observaron individuos solitarios con la única excepción de una hembra inmadura que se avistó durante varios días del mes de marzo en un roquedo de la Montaña Alavesa en el que crio una pareja en 1997. Se obtuvo una densidad global de 0,935 parejas/ 100 km², con una mayor concentración de territorios ocupados en los sectores este y oeste de la provincia (figura 1). Todas las parejas estuvieron asentadas en roquedos naturales ya que, aunque en años anteriores se había confirmado la reproducción de la especie en las paredes de tres canteras en desuso, ninguna de ellas estuvo ocupada en 2016.

\section{Continuidad de la ocupación de territorios}

El análisis para discriminar los territorios de ocupación continua $(n=20)$ de aquellos ocupados un solo año $(n=15)$ se llevó a cabo tras la exclusión de las variables que miden la distancia desde el núcleo de población más cercano a la pared de nidificación, y la altitud a la que esta se encuentra, fuertemente correlacionadas respectivamente con la distancia a la carretera más cercana $\left(r_{S}=0,782, n=35, P<0,001\right)$ y con la dominancia del roquedo $\left(r_{s}=0,738, n=35, P<0,001\right)$. Para obtener el modelo final se partió por lo tanto de las seis variables restantes, y tras el proceso de eliminación hacia atrás por pasos se retuvo como variable predictora únicamente a la dominancia, relacionada positivamente con la continuidad en la ocupación del territorio (tabla 1).

\begin{tabular}{|lcccc|}
\hline & Estima & Error est. & $z$ & $p$ \\
\hline (intercepto) & $-1,4954$ & 0,6819 & $-2,193$ & $0,0283^{*}$ \\
\hline dominancia & 0,0097 & 0,0034 & 2,876 & $0,0040^{* *}$ \\
\hline
\end{tabular}

Tabla 1.- Resultado del modelo lineal generalizado que relaciona la continuidad de ocupación de los territorios de halcón peregrino con las variables descriptoras del hábitat.

Table 1.- Results of the general linear model relating the continuity in the occupancy of peregrine falcon territories to several habitat variables. 


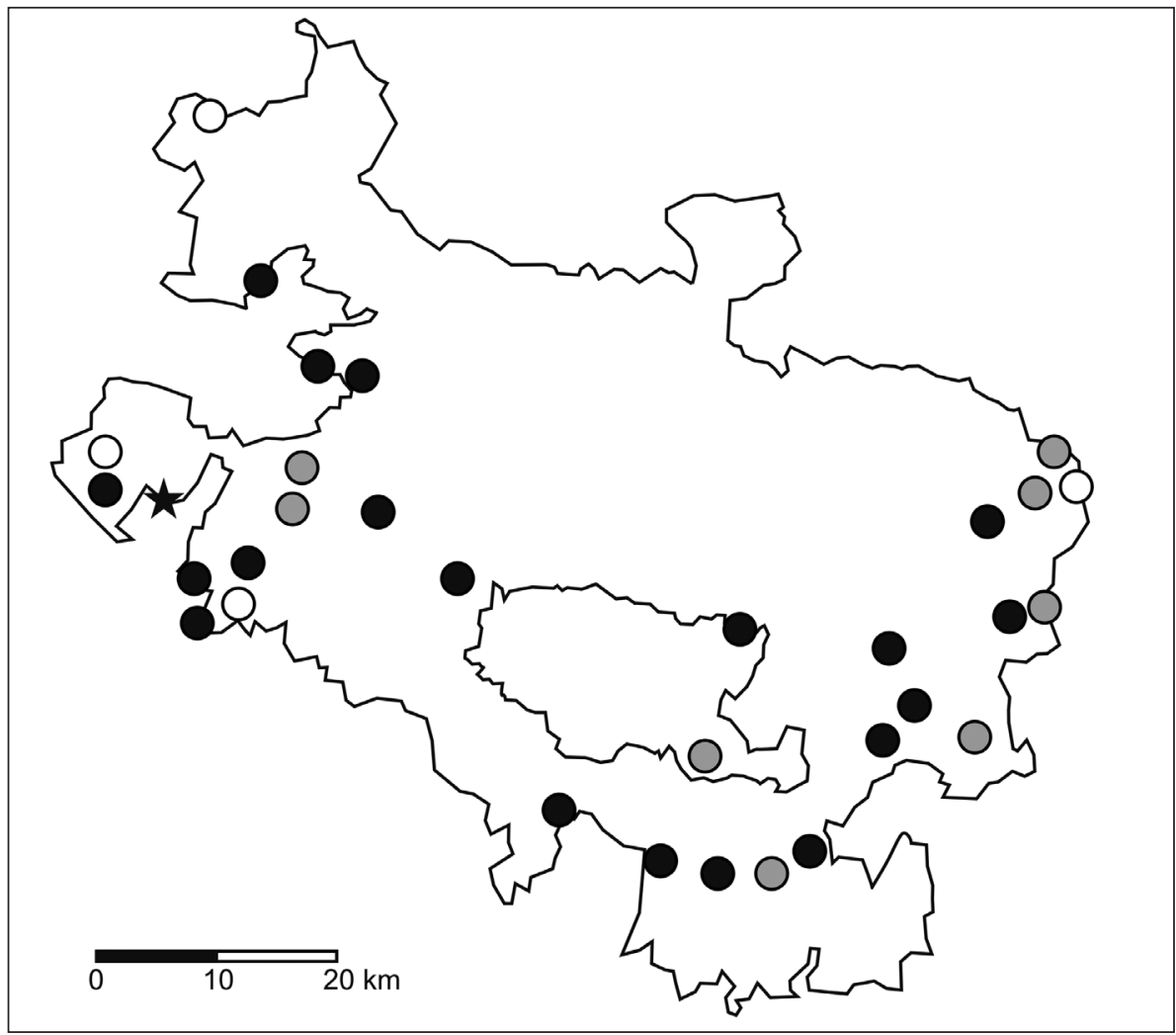

Fig. 1.- Ubicación de las parejas de halcón peregrino localizadas en el área de estudio en 2016. El color de los círculos refleja la continuidad de ocupación de los territorios en los censos provinciales de 1996/97, 2006/07 y 2016: territorios ocupados en los tres censos (negro), en dos de ellos (gris), y solo en 2016 (blanco). Con una estrella se señala un territorio a caballo entre Burgos y Álava, con presencia de la especie en los tres censos, pero no incluido en las cifras de 2016 por criar la especie este año en la parte burgalesa.

Fig. 1.- Geographical location of peregrine falcon pairs in the 2016 census. The colour of the circles shows the continuity in site occupancy according to the provincial censuses of 1996/97, 2006/07 and 2016: occupied territories in the three censuses (black), in two of them (grey), and only in 2016 (white). A star is used to indicate a territory on the border between Burgos and Álava, occupied in the three censuses, but not included in the 2016 results as the species bred in the Burgos sector.

\section{Tasas reproductoras}

De las veinte parejas seleccionadas al azar cuya reproducción se controló, quince criaron con éxito, sacando adelante un total de 28 pollos. Esto supone un éxito reproductor del 75\%, una tasa de vuelo de 1,87 pollos volados/ pareja con éxito, y una productividad de 1,40 pollos volados/ pareja territorial. En la figura 2 se presenta la evolución de la productividad en los seguimientos llevados a cabo en Álava hasta la 


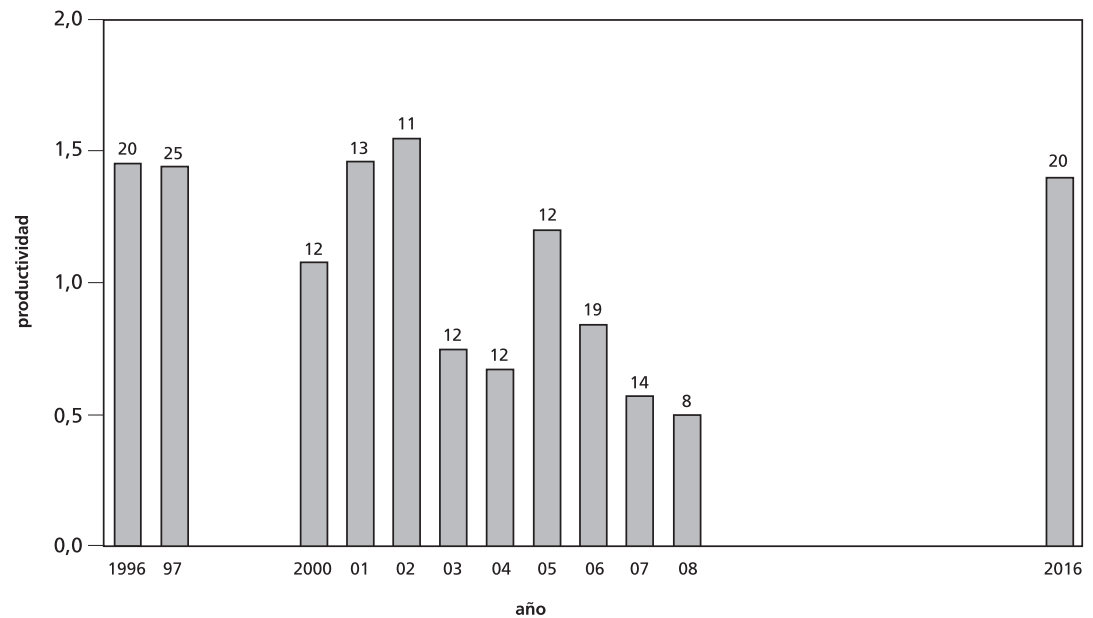

Fig. 2.- Evolución de la productividad (pollos volados/ pareja territorial) de una muestra de territorios de halcón peregrino en Álava. Sobre cada columna se indica el número de parejas cuya reproducción se siguió cada año.

Fig. 2.- Evolution of the productivity in a sample of peregrine falcon territories in Álava. The figures above each column show the number of pairs monitored each year.

fecha, observándose un claro aumento en la temporada 2016 tras el progresivo descenso del intervalo 1996-2008. La evolución de la productividad es paralela a la del éxito reproductor $\left(r_{s}=0,919, n=12, P<0,001\right)$. La tasa de vuelo por su parte ha permanecido mucho más constante en esta serie de años, con un valor máximo de 2,40 en 2005 y un mínimo de 1,87 en el presente censo de 2016.

Aunque se aprecia una cierta relación negativa entre la cantidad de lluvia en el mes de abril y los valores de productividad en las diferentes temporadas con datos, esta no es estadísticamente significativa $\left(r_{S}=-0,441, n=12, P=0,154\right)$.

\section{Edad de los componentes de la pareja}

Se pudo comprobar la edad de un total de 47 ejemplares de la especie, 24 machos y 23 hembras. Todos los machos eran adultos, pero cuatro de las hembras (17\%) eran individuos de segundo año calendario, es decir, nacidos en 2015 y con plumaje juvenil. Se siguió la reproducción de tres de estas parejas con hembra inmadura, de las cuales dos lograron sacar adelante dos pollos cada una. 


\section{Discusión}

\section{Tamaño y distribución de la población}

Con 31 parejas territoriales detectadas en 2016, la población alavesa de halcón peregrino presenta una densidad de 0,935 parejas / 100 km², un valor algo menor que el de las poblaciones vizcaína (Zuberogoitia et al., 2009) y guipuzcoana (Olano et al., 2018), y el más elevado de las provincias españolas interiores según los datos de 2008 recogidos en del Moral \& Molina (2009). Comparando los resultados de 2016 con las 31-33 parejas censadas en 1996/97 (Gainzarain et al., 2000) y las 31 de 2006/07 (del Moral \& Molina, 2009), los censos de los últimos veinte años muestran una marcada estabilidad de la población de la especie. Con anterioridad a estos censos, los datos disponibles sugieren un incremento poblacional a finales del siglo pasado, ya que en los primeros años noventa Illana (1991) contabiliza entre 21 y 26 parejas, y Lanius (1992), 24 parejas. No obstante, aunque es posible que este aumento tuviera lugar, los datos disponibles no permiten asegurarlo con certeza, ya que se trata en ambos casos de censos incompletos, que abordan respectivamente el estudio del halcón peregrino de forma secundaria con respecto a otras especies u otras provincias.

La estabilidad de la población alavesa en las últimas dos décadas coincide con la tendencia global que apuntan los resultados del censo nacional de 2008 para el conjunto de España, donde no obstante se dan marcados aumentos y disminuciones de efectivos en distintas provincias (del Moral \& Molina, 2009). Asimismo, se halla en la línea de la mayoría de las poblaciones europeas de la especie, que, una vez prohibidos los pesticidas organoclorados que causaron su declive a mediados del siglo pasado (Ratcliffe, 1993), atraviesan en la actualidad una fase de estabilidad o aumento (BirdLife International, 2015). En el caso del País Vasco, Olano et al. (2018) señalan asimismo una tendencia estable de la población guipuzcoana en las últimas dos décadas, tras el notable aumento detectado en los años 90, mientras que Zuberogoitia et al. (2009) encuentran una tendencia estable para la población vizcaína en los primeros años del presente siglo.

\section{Continuidad de la ocupación de territorios}

La estabilidad en las cifras totales no implica una constancia en la ocupación de los diferentes territorios del área de estudio. De hecho, entre uno y otro censo se observan numerosos cambios en la localización de las parejas nidificantes. El análisis llevado a cabo muestra que aquellos roquedos con una mayor continuidad en su ocupación tienden a estar situados en una situación de mayor dominancia sobre el entorno que los de ocupación más esporádica. La información en la que se basa el análisis corresponde a tres censos aislados en un periodo de veinte años, por lo que en realidad es un indicador indirecto de la continuidad de ocupación de un territorio determinado 
en este periodo. No obstante, la validez de los resultados obtenidos se ve reforzada por el hecho de que, en un análisis anterior en el que se compararon paredes con presencia de la especie con otras no ocupadas, Gainzarain et al. (2000) encontraron que la dominancia es la variable que determina en mayor medida la ocupación de un territorio. De este modo, esta variable parece tener un papel muy destacado en la selección de hábitat de nidificación de la especie en Álava, lo que coincide con lo observado por diversos autores tanto en Europa (Ratcliffe, 1993) como en Norteamérica (Bruggeman et al., 2015). El halcón peregrino buscaría así emplazamientos con una posición ventajosa desde la que descubrir presas e iniciar los vuelos de caza (Ratcliffe, 1993), más exitosos si se desarrollan desde un lugar elevado (Jenkins, 2000). Por otra parte, este tipo de paredes se encuentran más alejadas de la presencia humana que otras situadas en posiciones menos dominantes, y es posible que la menor frecuencia de perturbaciones también sea una condición favorable para el asentamiento de la especie (Rodríguez et al., 2007; Wilson et al., 2018).

\section{Tasas reproductoras}

En lo que respecta a las tasas reproductoras, las cifras de la población alavesa en 2016 son casi idénticas a las que del Moral \& Molina (2009) obtuvieron para el conjunto de España en el censo de 2008 (tasa de vuelo de 1,85 y productividad de 1,42). Comparando nuestros datos con los que recogen Rizzolli et al. (2005) para varias poblaciones europeas, se aprecia que, aunque la tasa de vuelo se encuentra cerca del límite inferior observado, la productividad muestra valores comparables a los recopilados por estos autores, y supera el umbral de un pollo volado por pareja territorial que se ha establecido en diversas ocasiones como el mínimo para una población en buen estado de conservación (Newton, 1979, USFWS, 2003). En relación con los resultados obtenidos en Álava en temporadas anteriores, se observa que la tasa de vuelo es algo inferior a lo que viene siendo habitual, pero la productividad se encuentra cerca de los valores máximos registrados.

La población alavesa de halcón peregrino presentó buenos valores de productividad en el periodo 1996-2002, año a partir del cual estos se redujeron sustancialmente. Alcanzaron valores mínimos en las dos últimas temporadas para las que se disponía de datos antes del presente censo (0,57 pollos/pareja territorial en 2007, y 0,50 en 2008), de manera que, del Moral \& Molina (2009), ya mencionan la amenaza que, a pesar de la estabilidad en el número de parejas nidificantes, suponían estos pobres resultados reproductores para el futuro de la población. Los datos del presente censo, aun con las precauciones que impone el hecho de que se refieran a una sola temporada, apuntan a que la tendencia a la baja no se ha agravado y que, al menos en 2016, la población alcanzó valores de productividad relativamente elevados. No contamos con información para determinar qué factores pueden explicar estas variaciones 
interanuales en el éxito de la reproducción, pero, como se ha demostrado en numerosas especies de aves (Newton, 1988), el mal tiempo durante la temporada de cría afecta negativamente a los parámetros reproductores, lo que también sucede en el caso del halcón peregrino (Mearns \& Newton, 1988; Ratcliffe, 1993; Zuberogoitia et al., 2002; Anctil et al., 2014). Zuberogoitia et al. (2015) encuentran que la precipitación total en abril es la variable que mejor explica las diferencias interanuales en la productividad de la especie en Vizcaya. Este es el mes en el que eclosionan los huevos y los pollos afrontan sus primeros días de vida, por lo que se encuentran más desprotegidos ante el frío y la humedad (Zuberogoitia et al., 2002). Si bien referidos a una corta serie de años, nuestros datos no muestran una relación significativa entre la productividad y la precipitación en abril, de modo que este factor no explica las grandes variaciones interanuales en el éxito reproductor de la población a lo largo de las dos últimas décadas.

\section{Edad de los componentes de la pareja}

El porcentaje observado del 17\% de hembras inmaduras es mucho mayor que el 3,7 \% obtenido en Vizcaya para un total de 272 intentos de reproducción estudiados durante once años (Zuberogoitia et al., 2009), aunque similar al observado en Guipúzcoa por Álvarez et al. (2005) en 2003 (12,5\%, n = 24) y 2004 (16,1\%, n= 31), así como al que Pandolfi et al. (2004) encuentran en 2001/02 en un área del sur de Italia $(19,7 \%)$. Esta elevada proporción de hembras inmaduras formando parte de la población nidificante podría indicar una alta mortalidad en el segmento reproductor de la población (Tordoff \& Redig, 1997), y contribuir además a unas bajas tasas reproductoras de la misma, ya que las hembras inmaduras suelen sacar adelante menos pollos que las adultas (Zabala \& Zuberogoitia, 2015). Pese a que la muestra en que se basa (23 ejemplares) es reducida y corresponde a una única temporada, y que dos de las tres parejas con hembras inmaduras cuya reproducción se controló fueron capaces de sacar sendas polladas de dos ejemplares, esta cifra de hembras jóvenes arroja una sombra sobre la aparente buena salud de la población alavesa de halcones y es un aspecto merecedor de un seguimiento adicional. Aunque la persecución directa de esta y otras especies de rapaces ha disminuido sustancialmente en los últimos tiempos en España (Martínez-Abraín et al., 2013; Martínez et al., 2016), los datos del Centro de Recuperación de Fauna Silvestre de Martioda, de la Diputación Foral de Álava, muestran que en la provincia la mortalidad por disparo aún tiene una incidencia apreciable, con cinco ingresos por esta causa en el decenio 2008-2017 (L. Elorza, com. pers.)

En conclusión, el censo de halcón peregrino en Álava en 2016 muestra una gran estabilidad de la población a lo largo de las últimas dos décadas, así como unos valores de productividad relativamente elevados. Estos resultados indican que la población se 
encuentra en un buen estado de conservación, si bien la alta proporción de hembras inmaduras podría ser síntoma de una elevada mortalidad adulta.

\section{Agradecimientos}

A Jesús Enjuto, Alfredo Conde, Andrés Illana e Îñigo Zuberogoitia, que amablemente nos comunicaron diversas observaciones de halcón peregrino. A Laura Elorza, del Centro de Recuperación de Fauna Silvestre de Martioda, de la Diputación Foral de Álava, por su información sobre ingresos de la especie. A Íñigo Zuberogoitia, Pablo Vera y Emilio Barba, por sus valiosos comentarios y sugerencias que mejoraron sustancialmente una primera versión del manuscrito. La Sección de Espacios Naturales y Biodiversidad de la Diputación Foral de Álava financió los gastos de desplazamiento del presente estudio.

\section{Bibliografía}

Álvarez, J., Bea, A., Faus, J M., Castién, E., Mendiola, I. 1985. Atlas de los vertebrados continentales de Álava, Vizcaya y Guipúzcoa (exc. Chiroptera). Gobierno Vasco. Vitoria- Gasteiz.

Álvarez, F., Olano, M., Aierbe, T., Vázquez, J., Izkeaga, P., Ugarte, J. 2005. Población, distribución y reproducción del Halcón peregrino (Falco peregrinus) en Gipuzkoa (2003-2004). Munibe, Cienc. Nat. 56: 113-132.

Anctil, A., Franke, A., Bêty, J. 2014. Heavy rainfall increases nestling mortality of an arctic top predator: experimental evidence and long-term trend in peregrine falcons. Oecologia 174: 1033-1043.

BirdLife International. 2015. European Red List of Birds. Office for Official Publications of the European Communities. Luxemburgo.

Bruggeman, J.E., Swem, T., Andersen, D. E., Kennedy, P.L., Nigro, D. 2015. Multi-season occupancy models identify biotic and abiotic factors influencing a recovering Arctic Peregrine Falcon Falco peregrinus tundrius population. Ibis 158: 61-74.

Cade, T.J., Enderson, J.H., Thelander, C.G., White, C.M. (eds.) 1988. Peregrine Falcon Populations: Their Management and Recovery. The Peregrine Fund Inc. Boise, Idaho.

Del Moral, J.C., Molina, B. (eds.) 2009. El halcón peregrino en España. Población reproductora en 2008 y método de censo. SEO/BirdLife. Madrid.

Dormann, C.F., Elith, J., Bacher, S., Buchman, C., Carl, G., Carré, G., García Márquez, J.R., Gruber, B., Lafourcade, B., Leitão, P.J., Münkemüller, T., McClean, C., Osborne, P.E., Reineking, B., Schröder, B., Skidmore, A.K., Zurell, D., Lautenbach, S. 2013. Collinearity: a review of methods to deal with it and a simulation study evaluating their performance. Ecography 36: 27-46. 
Esri. 2016. ArcGIS Desktop: Release 10.4.1. Environmental Systems Research Institute. Redlands.

Gainzarain, J.A., Arambarri, R., Rodríguez, A.F. 2000. Breeding density, habitat selection and reproductive rates of the Peregrine Falcon Falco peregrinus in Álava (N Spain). Bird Study 47: 225-231.

Gainzarain, J.A., Arambarri, R., Rodríguez, A.F. 2002. Population size and factors affecting the density of the Peregrine Falcon Falco peregrinus in Spain. Ardeola 49: 67-74.

Hardey, J., Crick, H., Wernham, C., Riley, H., Etheridge, B., Thompson, D. 2009. Raptors: a field guide for surveys and monitoring. Stationery Office. Edimburgo.

Heredia, B., Hiraldo, F., González, L.M, González, J.L. 1988. Status, ecology and conservation of the Peregrine Falcon in Spain. En, Peregrine Falcon Populations: Their Management and Recovery. T. J. Cade, J. H. Enderson, C. G. Thelander, C. M. White (Eds.), pp. 219-226. The Peregrine Fund Inc. Boise. Idaho.

Horne G., Fielding, A.H. 2002. Recovery of the Peregrine Falcon Falco peregrinus in Cumbria, UK, 1966-99. Bird Study 49: 229-236.

Illana, A. 1991. Rapaces rupícolas diurnas de Álava. Diputación Foral de Álava. Vitoria-Gasteiz.

Jenkins, A.R. 2000. Hunting mode and success of African Peregrines Falco peregrinus minor: does nesting habitat quality affect foraging efficiency? Ibis 142: 235-246.

Lanius. 1992. Censo de Halcón Común de la Comunidad Autónoma Vasca. Año 1991. Informe inédito.

Martínez, J.E., Zuberogoitia, I., Jiménez-Franco, M.V., Calvo, J.F. 2016. Spatio-temporal variations in mortality causes of two migratory forest raptors in Spain. Eur. J. Wildl. Res. 62: 109-118.

Martínez-Abraín, A., Crespo, J., Berdugo, M., Gutiérrez, L., Lafuente, A., Mañas, A., de Miguel, J.M. 2013. Causes of human impact to protected vertebrate wildlife parallel long-term socio-economical changes in Spain. An. Conserv. 16: 286-294.

Mearns, R., Newton, I. 1998. Factors affecting breeding success of Peregrines in South Scotland. J. Animal Ecol. 57: 903-916.

Newton, I. 1979. Population Ecology of Raptors. T \& AD Poyser. Berkhamsted.

Newton, I. 1998. Population Limitation in Birds. Academic Press. Londres.

Olano, M., Ansorregi, F., Aierbe, T., Hurtado, R., Vázquez, J., Ugarte, J., Beñaran, H., Galdós, A., Arizaga, J. 2018. Distribution and demographic parameters of the Peregrine falcon Falco peregrinus L., 1756 in Gipuzkoa. Munibe, Cienc. nat. 66 (en prensa).

Pandolfi, M., Gaibani, G., Tanferna, A. 2004. Depicts the number of breeding pairs reliably the status of Peregrine Falcon Falco peregrinus? Ardea 92: 247-251.

R Core Team. 2018. R. A language and environment for statistical computing. R Foundation for Statistical Computing, Vienna, Austria. Available at: https://www.R-project.org/. 
Ratcliffe, D.A. 1993. The Peregrine Falcon. Poyser. Londres.

Rizzolli, F., Sergio, F., Marchesi, L., Pedrini, P. 2005. Density, productivity, diet and population status of the Peregrine Falcon Falco peregrinus in the Italian Alps. Bird Study 52: 188-192.

Rodríguez, B., Siverio, M., Rodríguez, A., Siverio, F. 2007. Density, habitat selection and breeding success of an insular population of Barbary Falcon Falco peregrinus pelegrinoides. Ardea 95: 213-223.

Sergio, F., Rizzolli, F., Marchesi, L., Pedrini, P. 2004. The importance of interspecific interactions for breeding-site selection: Peregrine falcons seek proximity to Raven nests. Ecography 27: 818-826.

Sielicki, J., Mizera, T. 2009. Peregrine Falcon populations -status and perspectives in the 21st century. Turul Publishing and Poznán University of Life Sciences Press. Varsovia.

Tellería, J. L. 2012. Introducción a la conservación de las especies. Tundra Ediciones. Valencia.

Tordoff, H.B., Redig, P. T. 1997. Midwest Peregrine Falcon demography, 1982-1995. J. Raptor Res. 31: 339-346.

U.S. Fish and Wildlife Service. 2003. Monitoring plan for the American Peregrine Falcon, a species recovered under the Endangered Species Act. U.S. Fish and Wildlife Service, Divisions of Endangered Species and Migratory Birds and State Programs, Pacific Region, Portland, OR, USA.

Wightman, C.S., Fuller, M.R. 2005. Spacing and habitat selection patterns of Peregrine Falcons in central West Greenland. The Wilson Bull. 117, 226-236.

Wilson, M.W., Balmer, D.E., Jones, K., King, V.A., Raw, D., Rollie, C.J., Rooney, E., Ruddock, M., Smith, G.D., Stevenson, A., Stirling-Aird, P.K., Wernham, C.V., Weston, J. M., Noble, D.G. 2018. The breeding population of Peregrine Falcon Falco peregrinus in the United Kingdom, Isle of Mann and Channel Islands in 2014. Bird Study 65: 1-19.

Zabala, J., Zuberogoitia, I. 2015. Breeding performance and survival in the peregrine falcon Falco peregrinus support an age-related competence improvement hypothesis mediated via an age threshold. J. Avian Biol. 45: 141-150

Zuberogoitia, I. 1997. Seguimiento de la población de halcones peregrinos en Bizkaia $1^{\mathrm{er}}$ año (1997). Munibe, Cienc. nat. 49: 111-116.

Zuberogoitia, I., Ruiz Moneo, J. F., Torres, J. J. 2002. El Halcón Peregrino. Diputación Foral de Bizkaia. Bilbao.

Zuberogoitia, I., Ruiz Moneo, J. F., Torres, J. J. 2002. El Halcón Peregrino. Diputación Foral de Bizkaia. Bilbao.

Zuberogoitia, I., Martínez, J. A., Azkona, A., Martínez, J. E., Castillo, I., Zabala, J. 2009. Using recruitment age, territorial fidelity and dispersal as decisive tools in the conservation and management of peregrine falcon (Falco peregrinus) populations: the case of a healthy population in northern Spain. J. Ornithol. 150: 95-101. 
Gainzarain et al.

Zuberogoitia, I., Zabala, J., Martínez, J. E., Olsen, J. 2015. Alternative eyrie use in peregrine falcons: is it a female choice? J. Zool. 296: 6-14.

Zuur, A. F., leno, E. N., Smith, G M. 2007. Analysing Ecological Data. Springer. Nueva York.

Fecha de recepción/Date of reception: 20/12/2017

Fecha de aceptación/Date of acceptance: 29/08/2018

Editor Asociado/Associate editor: Emilio Barba 